\title{
A characterization of generalized Rudin-Shapiro sequences with values in a locally compact abelian group
}

by

\author{
Jean-Loup Mauclaire (Paris)
}

1. Introduction. Let $G$ be a locally compact abelian group with a group law denoted additively. Let $A$ be a finite alphabet $\{0,1,2, \ldots, q-1\}$ of $q$ elements naturally ordered, with a fixed integer $q \geq 2$. We denote by $A^{*}$ the monoid of finite words on $A$. The number of letters of an element $w$ of $A^{*}$ is the length of the word, and with every word $w=w_{0} w_{1} \ldots w_{r}$ of length $r+1$, we associate the integer $\dot{w}=w_{0}+q w_{1}+\ldots+q^{r} w_{r}$. Following J.-P. Allouche and P. Liardet (see [1]), we shall say that a map $f$ from $A^{*}$ to $G$ is a chained map if it satisfies the condition that for all letters $a$ and $b$ in $A$, and any word $w$ in $A^{*}, f(a b w)=f(a b)-f(b)+f(b w)$. Note that, by iteration, for all letters $a_{1}, a_{2}, \ldots, a_{s}$, and every word $w$ in $A^{*}$, we have

$$
f\left(a_{1} a_{2} \ldots a_{s} w\right)=\left(f\left(a_{1} a_{2}\right)-f\left(a_{2}\right)\right)+\ldots+\left(f\left(a_{s-1} a_{s}\right)-f\left(a_{s}\right)\right)+f\left(a_{s} w\right) .
$$

Now, given a chained map $f$, if $\chi$ is a continuous character of $G$, we denote by $\chi T$ the $q^{2}$-matrix with entries $a_{i}^{j}=\chi(f(i j)-f(j)), 0 \leq j \leq q-1$, $0 \leq i \leq q-1$, where $a_{i}^{j}$ is the element of the $i$ th line and $j$ th column. By definition, $f$ is said to be a Rudin-Shapiro map if for every non-trivial character $\chi,(\overline{\chi T})(\chi T)=q I$, or equivalently $(\chi T)(\overline{\chi T})=q I$. Here $I$ denotes the identity matrix and $(\overline{\chi T})$ the adjoint of $(\chi T)$. With every $f$ we associate a sequence $f^{*}$ defined by $f^{*}(\dot{w})=f(w)$. Such a sequence $f^{*}$ will be called a generalized Rudin-Shapiro sequence with values in $G$ if $f$ is a Rudin-Shapiro map.

In [1], motivations are given for the study of these sequences, which generalize in a natural way the classical Rudin-Shapiro sequence. But the authors assume that $G$ is compact and metrizable. In this paper we consider the more general case where $G$ is a locally compact abelian group. One of the interesting results presented in [1] is that if $G$ is a compact metrizable abelian group and if $f$ is a Rudin-Shapiro map, then $G$ must be finite. The proof given in [1] is not quite correct. The purpose of this article is to give a complete proof for the more general case of locally compact abelian 
groups. Moreover, we give a characterization of the existence of generalized Rudin-Shapiro sequences with values in a locally compact abelian group.

2. The result. We shall give a proof of the following theorem:

Theorem. Let $G$ be a locally compact abelian group written additively. $A$ mapping $f$ is a Rudin-Shapiro map from $A^{*}$ to $G$ if and only if

(i) $G$ is a finite group, and its order $N$ divides $q$,

(ii) if $u_{i j}(r)=f(i r)-f(j r), 0 \leq i, j, r \leq q-1$, then for every $\varrho$ in $G$ and every $i \neq j$, the number $N_{i j}(\varrho)=\operatorname{card}\left\{u_{i j}(r): 0 \leq r \leq q-1, u_{i j}(r)=\varrho\right\}$ is equal to $q / N$.

Remark. Property (ii) is equivalent to the fact that the set of the differences $f(i r)-f(j r), 0 \leq r \leq q-1, i \neq j$, is the union of $q / N$ copies of $G$.

\section{Proof of the Theorem}

I. We assume that $f$ is a Rudin-Shapiro map. Let $\chi$ be a non-trivial character of $G$. Consider $\chi T$, the $q \times q$ matrix with entries

$$
a_{i}^{j}=\chi(f(i j)-f(j)), \quad 0 \leq j \leq q-1,0 \leq i \leq q-1 .
$$

We have $\chi T=(\chi(f(i j)))(\bar{\chi}(f(j)))$, where $(\chi(f(i j)))$ (resp. $(\bar{\chi}(f(j)))$ is the $q \times q$ matrix with entries $\widetilde{a}_{i}^{j}=\chi(f(i j))$ (resp. $\widehat{a}_{i}^{j}=\bar{\chi}(f(j))$ if $i=j$ and $\widehat{a}_{i}^{j}=0$ otherwise). Now, since $f$ is a Rudin-Shapiro map, we have $(\chi T)(\overline{\chi T})=q I$, which implies $(\chi(f(i j)))(\overline{\chi(f(i j))})=q I$ and gives

$$
\sum_{r=0}^{q-1} \chi(f(i r)-f(j r))= \begin{cases}q & \text { if } i=j, \\ 0 & \text { if } i \neq j .\end{cases}
$$

Now, we fix $i$ and $j, i \neq j$, and set $u(r)=f(i r)-f(j r), 0 \leq r \leq q-1$. Since $G$ is locally compact, we consider a compact neighbourhood $V(0)$ of the origin. Let $F$ be a continuous non-negative real function with support in $V(0)$ taking the value 1 at 0 and such that its Fourier transform $F^{*}$ is invertible. The function $H$ defined by

$$
H(t)=\sum_{r=0}^{q-1} F(u(r)+t)
$$

has a Fourier transform $H^{*}$ given by

$$
H^{*}(\chi)=\sum_{r=0}^{q-1} \int_{G} F(u(r)+t) \chi(t) d m(t)
$$


where $m$ is the normalized Haar measure on $G$. Therefore

$$
H^{*}(\chi)=\sum_{r=0}^{q-1} \bar{\chi}(u(r)) \int_{G} F(u(r)+t) \chi(t+u(r)) d m(t)
$$

and so

$$
H^{*}(\chi)=F^{*}(\chi) \sum_{r=0}^{q-1} \bar{\chi}(u(r)) .
$$

Hence $H^{*}(\chi)$ is equal to zero, except if $\chi$ is trivial. Denoting by $m^{*}$ the normalized Haar measure on $G^{*}$, the dual group of $G$, by Fourier inversion we get

$$
\begin{aligned}
\int_{G^{*}} H^{*}(\chi) \bar{\chi}(t) d m^{*}(\chi) & =\int_{G^{*}} F^{*}(\chi) \sum_{r=0}^{q-1} \bar{\chi}(u(r)) \bar{\chi}(t) d m^{*}(\chi) \\
& =\sum_{r=0}^{q-1} F(u(r)+t)=H(t) .
\end{aligned}
$$

Since $H$ is not identically 0 by construction, we deduce that if $1_{G^{*}}$ denotes the trivial character on $G$, then $m^{*}\left(\left\{1_{G^{*}}\right\}\right)$ is not 0 and is finite since a point is compact. But $m^{*}$ is translation invariant so that $m^{*}(\{\chi\})=m^{*}\left(\left\{1_{G^{*}}\right\}\right)$ for any character $\chi$ of $G$. Now, any compact neighbourhood $V^{*}$ of $1_{G^{*}}$ has a finite measure and so must be a finite set. Indeed, if $\left\{\chi_{m}: 0 \leq m \leq n\right\}$ is a set of distinct elements of $V^{*}$, we must have

$$
\sum_{m=0}^{n} m^{*}\left(\left\{\chi_{m}\right\}\right)=(n+1) m^{*}\left(\left\{1_{G^{*}}\right\}\right)<m^{*}\left(V^{*}\right),
$$

which implies that $n$ is finite. This proves that the topology defined on $G^{*}$ is discrete. By a classical result of duality theory, we deduce that $G$ is compact. Now, we consider a finite family of compact neighbourhoods $V(r)$ of $u(r), 0 \leq r \leq q-1$, and assume that there exists a continuous non-negative real function $F$, not identically 0 , with compact support $S(F)$ such that $S(F)$ does not intersect any of the $V(r)$. Since $G$ is compact, the space $E$ of finite linear combinations of characters of $G$ is dense in the space of continuous functions on $G$. Then, for any given $\varepsilon>0$, there exists an approximation $P_{\varepsilon} \in E$ such that $\sup _{t \in G}\left|F(t)-P_{\varepsilon}(t)\right| \leq \varepsilon$. Note that $\left|\int_{G}\left(F-P_{\varepsilon}\right) d m\right| \leq \varepsilon$, and recall that $\widehat{P}_{\varepsilon}\left(1_{G^{*}}\right)=\int_{G} P_{\varepsilon} d m, \widehat{P}_{\varepsilon}\left(1_{G^{*}}\right)$ denoting the Fourier transform of $P_{\varepsilon}$ at $1_{G^{*}}$. But $\sup _{t \in G}\left|F(t)-P_{\varepsilon}(t)\right| \leq \varepsilon$ also implies that $\left|\sum_{r=0}^{q-1} F(u(r))-P_{\varepsilon}(u(r))\right| \leq q \varepsilon$ and since $F(u(r))=0$ for every $r$ and $\sum_{r=0}^{q-1} \chi(u(r))=0$ for every non-trivial character $\chi$ of $G$, we get $\left|\sum_{r=0}^{q-1} \widehat{P}_{\varepsilon}\left(1_{G^{*}}\right)\right| \leq q \varepsilon$. Therefore $\int_{G} F=0$. Since $F$ is continuous, nonnegative and not identically 0 , this is a contradiction. Hence $S(F)$ intersects 
at least one of the compact neighbourhoods $V(r)$. This means that the finite set of points $u(r), 0 \leq r \leq q-1$, is dense in $G$, and so $G$ is finite.

We shall denote by $N$ the number of elements of $G$.

By our hypothesis, $\sum_{r=0}^{q-1} \chi(u(r))=0$ for every non-trivial character $\chi$ of $G$. Now, we write

$$
\sum_{r=0}^{q-1} \chi(u(r))=\sum_{\varrho \in G} \chi(\varrho) N(\varrho)
$$

where, as above, $N(\varrho)=\operatorname{card}\{u(r): 0 \leq r \leq q-1, u(r)=\varrho\}$. For $\varrho$ in $G$, the orthogonality relations for characters give

$$
N(\varrho)=\frac{1}{N} \sum_{\chi \in G^{*}} \bar{\chi}(\varrho)\left(\sum_{\varrho^{\prime} \in G} \chi\left(\varrho^{\prime}\right) N\left(\varrho^{\prime}\right)\right) .
$$

Using (1), we see that formula (2) can be written

$$
N(\varrho)=\frac{1}{N} \sum_{\chi \in G^{*}} \bar{\chi}(\varrho)\left(\sum_{r=0}^{q-1} \chi(u(r))\right),
$$

and since by hypothesis $\sum_{r=0}^{q-1} \chi(u(r))$ is 0 if $\chi \neq 1$ and $q$ if $\chi=1$, we get $N(\varrho)=q / N$. In particular, $N$ divides $q$ since $q / N$ is an integer.

So, we have shown that

(i) $G$ is a finite group and its order $N$ divides $q$,

(ii) if we fix $i$ and $j, i \neq j$, and set $u_{i j}(r)=f(i r)-f(j r), 0 \leq r \leq q-1$, then for every $\varrho$ in $G$,

$$
N_{i j}(\varrho)=\operatorname{card}\left\{u_{i j}(r): 0 \leq r \leq q-1, u_{i j}(r)=\varrho\right\}=q / N .
$$

II. We prove the converse. To this end, we assume that $G$ is a finite group and its order $N$ divides $q$. Let $f$ be a chained map satisfying the following property:

For any $i$ and $j$ such that $i \neq j$, the set of the differences $f(i r)-$ $f(j r), 0 \leq r \leq q-1$, is the union of $q / N$ copies of $G$.

We shall prove that if $\chi$ is a non-trivial character of $G$, then $\chi T$, the $q \times q$ matrix with entries $a_{i}^{j}=\chi(f(i j)-f(j))$, satisfies $(\chi T)(\overline{\chi T})=q I$.

We have seen that $\chi T=(\chi(f(i j)))(\bar{\chi}(f(j)))$. Note that $(\chi T)(\overline{\chi T})=$ $\left(A_{i}^{j}\right)$ with

$$
\begin{aligned}
A_{i}^{j} & =\sum_{r=0}^{q-1} \chi(f(i r)) \bar{\chi}(r) \overline{\bar{\chi}}(r) \bar{\chi}(f(j r))=\sum_{r=0}^{q-1} \chi(f(i r)) \bar{\chi}(f(j r)) \\
& =\sum_{r=0}^{q-1} \chi(f(i r)-f(j r)) .
\end{aligned}
$$


By assumption,

$$
\sum_{r=0}^{q-1} \chi(f(i r)-f(j r))=\sum_{\varrho \in G} \frac{q}{N} \chi(\varrho)=\frac{q}{N} \sum_{\varrho \in G} \chi(\varrho) .
$$

Therefore $A_{i}^{j}=q$ if $i=j$ and $A_{i}^{j}=0$ if $i \neq j$. This means that $(\chi T)(\overline{\chi T})$ $=q I$, i.e., $f$ is a Rudin-Shapiro map. This ends the proof of the Theorem.

EXAmPles. 1 . In the case $q=2, G=\mathbb{Z} / 2 \mathbb{Z}$, the classical Rudin-Shapiro sequence is obtained by taking $f(i j)$ equal to the coefficient of the $i$ th line and $j$ th column of the $2 \times 2$ matrix $\left[\begin{array}{rr}1 & 1 \\ 1 & -1\end{array}\right]$ ([3], [4]).

2. Let $q$ be a prime number. We identify $A$ with the cyclic group of order $q$. Then, as mentioned in [1] (where the condition on the primality of $q$ has been omitted), the example given by Queffélec in [2] corresponds, in this case, to the $A$-valued Rudin-Shapiro map $f$ defined on $A \times A$ by $f(a, b)=a b$.

\section{References}

[1] J.-P. Allouche and P. Liardet, Generalized Rudin-Shapiro sequences, Acta Arith. 60 (1991), 1-27.

[2] M. Queffélec, Une nouvelle propriété des suites de Rudin-Shapiro, Ann. Inst. Fourier (Grenoble) 37 (2) (1987), 115-138.

[3] W. Rudin, Some theorems on Fourier coefficients, Proc. Amer. Math. Soc. 10 (1959), 855-859

[4] H. S. Shapiro, Extremal problems for polynomials and power series, Thesis, M.I.T., 1951.

C.N.R.S., U.R.A. 212

THÉORIES GÉOMÉTRIQUES

UNIVERSITÉ PARIS-VII

TOUR $\mathrm{N}^{\circ} 45-55,5^{\mathrm{e}}$ ÉTAGE

2, PLACE JUSSIEU

75251 PARIS CEDEX 05

FRANCE 